\title{
Chemo-resistant gestational trophoblastic neoplasia, 5-years experience of Mansoura University Hospital, Egypt
}

\author{
Reda Abd Elhady Hemida ${ }^{1 *}$, Eman Toson ${ }^{2}$, Hend Shalaby ${ }^{1}$, Ehsan Refaie ${ }^{1}$, Doaa Sharaf Eldin ${ }^{2}$ \\ ${ }^{1}$ Department of Obstetrics and Gynaecology, Mansoura University Hospital, Mansoura, Egypt. \\ ${ }^{2}$ Department of Clinical Oncology, Mansoura University Hospital, Mansoura, Egypt. \\ E-mail: *redaelshouky@hotmail.com
}

Received 25 April 2011; revised 23 May 2011; accepted 30 May 2011.

\begin{abstract}
Introduction: Gestational trophoblastic neoplasia (GTN), is recognized as the most curable gynaecologic malignancy. However, many cases are resistant to first line chemotherapy. Objective: The aim of the study is to report our 5 years experience in the management of GTN cases with special stress on the chemo-resistant cases. Methods: The study was performed through reviewing the records of 51 patients who were diagnosed as GTN during the period from 1/1/2006 to 31/12/2010 in Mansoura University Hospital, Egypt. Results: Resistance to methotrexate therapy was reported in $15.15 \%$ of low risk cases and received etoposide or cisplatinum/etoposide. Sixty percent of high risk cases were resistant to MAC combination and received salvage chemotherapy or hysterectomy. There was significant correlation between patient response and initial B-hCG, as well as WHO risk score ( $P$ value $=0.001$ in both) but correlations with age, parity, type of antecedent pregnancy, and histopathology were non significant $(p=0.95$, $0.53,0.47 \& 0.83$ respectively). Conclusion: Low risk GTN cases who were resistant to methotrexate monotherapy received etoposide or cisplatinum/etoposide as a second-line therapy. High risk GTN cases who were resistant to MAC combination received secondline combination chemotherapy and/or hysterectomy. WHO risk score and initial B-hCG were correlated to resistance to first line chemotherapy.
\end{abstract}

Keywords: Gestational Trophoblastic NeoplasiaChemotherapy-Resistance

\section{INTRODUCTION}

Gestational trophoblastic neoplasia (GTN), is the term now commonly applied to persistent or invasive gestational trophoblastic disease [1]. GTN is typically diag- nosed in asymptomatic women undergoing routine hCG monitoring after evacuation of a complete molar pregnancy. It is recognized today as the most curable gynaecologic malignancy [1]. The reported incidence of GTN is 2/1000 pregnancies in Japan and $0.6-1.1 / 1000$ pregnancies in Europe [2]. In Africa, Moodley and colleagues reported that the incidence of GTN was $0.5 / 1000$ deliveries in South Africa [3].

There are many classifications of GTN, in 1973, Hammond et al. classified GTN into non metastatic and metastatic diseases, the later is further subdivided into good prognosis metastatic disease and poor prognosis metastatic disease. In 1976, Bagshawe suggested the use of a prognostic scoring system. The WHO has adopted a modification of Bagshawe's scoring system [1].

Patients scored as low risk (score 0 - 6) receive methotrexate and folinic acid and have a survival rate of about $100 \%$, but a third require second-line chemotherapy, either with single-agent intravenous actinomycin $\mathrm{D}$ or with etoposide, methotrexate, actinomycin D alternating with cyclophosphomide and vincristine (EMA/CO) [4-6].

Patients scored as high risk (score $\geq 7$ ) receive EMA/ $\mathrm{CO}$ as first-line therapy and have a survival rate of $90 \%$ but more than $10 \%$ of patients fail this therapy $[6,7]$. To salvage women failing EMA/CO, Newlands et al. [8] have previously shown that the addition of cisplatin to etoposide (EP) alternating weekly with EMA (1 day only) with or without surgery salvages a further $75 \%$ of cases. El-lamie et al. [9], recommended incorporation of paclitaxel in the third-line treatment of resistant GTN. However, Deng et al. [10], concluded from a Cochrane systematic review that a MAC regimen was better than other regimens for high-risk GTN because of lower toxicity.

Furthermore, adjuvant surgical procedures could be excellent adjuncts to salvage chemotherapy in removing known foci of chemotherapy-resistant disease in selected patients with persistent GTN [11].

Zhou et al. [12], classified refractory GTN as chemo- 
resistant GTN group who had never a normal serum beta subunit of human chorionic gonadotropin $(\beta$-hCG) level during their previous treatment, relapsed GTN group who had elevated serum $\beta$-hCG levels in the absence of the pregnancy after finished treatment 3 months or more, and undetermined GTN group who had elevated serum $\beta$-hCG levels in the absence of the pregnancy less than 3 months after completed treatment. They concluded that comparing with the patients with chemo-resistant GTN, the outcome of patients with relapsed GTN was better.

Optimization of treatment strategies for patients who develop drug resistance remains a key challenge. We tried to clarify factors that were linked to resistance to first-line chemotherapy and management of the chemoresistant cases during the last 5 years in our university hospital

\section{PATIENTS AND METHODS}

The study was performed through reviewing the records of 51 patients who were diagnosed as gestational trophoblastic neoplasia during the period from 1/1/2006 to $31 / 12 / 2010$ in the departments of Gynaecology and Clinical Oncology, Mansoura University Hospital, Egypt.

The cases were diagnosed as GTN after persistent positive hCG more than 6 months, plateuing, or rising serum level of B-hCG after evacuation of molar pregnancy or after histological diagnosis of choriocarcinoma, invasive mole, or placental site trophoblastic tumour (PSTT).

The patients were evaluated with respect to age, parity, type of antecedent pregnancy, clinical presentation, and presence of metastasis. The level of serum B-subunit of hCG at diagnosis, and its level on follow up visits were studied. Uterine re-curettage and pathological findings were evaluated. All women were classified as low-risk or high-risk disease using FIGO and WHO scoring systems [1].

The first line chemotherapy treatment for these cases was studied regarding its type (single or multiple agent), number of courses, and the response rate. Patients needed second, third lines of combination chemotherapy as well as performing hysterectomy or other surgical procedures were also analyzed.

\section{STATISTICAL ANALYSIS}

The statistical analysis of data done by using excel program for figures and SPSS program (SPSS, Inc, Chicago, USA) statistical package for social science version 16 .

To test the normality of data distribution K-S (Kolmogorov-Smirnov) test was done only significant data revealed to be nonparametric. The description of the data done in form of mean (+/-) SD for quantitative data and Frequency \& proportion for qualitative data.
The analysis of the data was done to test statistical significant difference between groups. For quantitative date student t-test was used to compare between two groups.

Paired sample t-test was used to compare one group at different times. Chi square test was used for for qualitative data. $\mathrm{P}$ is considered significant if less than or equal to 0.05 at confidence interval $95 \%$.

\section{RESULTS}

This retrospective study included 51 cases who were diagnosed as GTN. The mean follow up duration was 28.45 months (range: 3 - 54). Thirty-six cases $(70.6 \%)$ were low risk and 15 cases (29.4\%) high risk according to WHO scoring system. The mean age was 28.7 years (range: 17 - 50 years). The mean parity was 1.29 (range: 0 - 5). Mean B-hCG level on admission was 52705.8 $\mathrm{mu} / \mathrm{ml}$ (range: 60.0 - 500,000). The mean time since the last pregnancy was 6.15 months (range: 1 - 72).

The history of the previous pregnancy was shown in Table 1, as can be seen from the table, 30 cases $(58.8 \%)$, followed complete hydatidiform mole.

The initial clinical presentation of the studied cases was post molar bleeding in 24 cases (47.05\%), nonnormalization of hCG after molar evacuation in 10 cases (19.60\%), abnormal vaginal bleeding in 16 cases $(31.38 \%)$, and one case $(1.97 \%)$, was presented to the emergency unit by internal haemorrhage with elevated hCG (1000 $\mathrm{mu} / \mathrm{ml}$ ) and diagnosed after laparotomy as ruptured hepatic metastasis of choriocarcinoma .

Dilatation and curettage was done in 36 cases $(70.6 \%)$, the histopathology of these cases was shown in Table 2. There were 8 cases $(15.68 \%)$ with distant metastases, the sites of metastasis are shown in Table 3.

Forty-eight patients received first line chemotherapy for 2 - 15 cycles, 3 low risk cases (5.9\%) did not receive chemotherapy due to spontaneous decline of B-hCG after uterine re-curettage (a total of 51 cases). Complete response was achieved in 34 patients $(70.83 \%)$, while 14 cases were resistant to chemotherapy $(29.17 \%)$.

Regarding first- line treatment, 33 low-risk cases received methotrexate in a dose of $1 \mathrm{mg} / \mathrm{kg}$ in days $1,3,5$, and 7 alternating with oral folinic acid $0.1 \mathrm{mg} / \mathrm{kg}$ in days 2, 4, 6, and 8. Mean number of courses was 5.09 (range: 2 - 15). Twenty-eight cases $(84.85 \%)$ achieved remission, while 5 cases $(15.15 \%)$ needed second line therapy in the form of etoposide (3 cases) and cisplatinum/etoposide ( 2 cases) with a complete response.

High risk patients (15 cases) received methotrexate actinomycin D-cyclophosphamide (MAC) combination as first-line chemotherapy, 6 cases $(40 \%)$ responded to this treatment while 9 cases $(60 \%)$ were resistant. According to our tumour discussion panel, 6 of the resistant cases received cisplatinum/etoposide as second-line 
Table 1. The type of antecedent pregnancy.

\begin{tabular}{ccc}
\hline & Count & Percentage \\
\hline Abortion & 10 & $19.6 \%$ \\
Partial mole & 9 & $17.6 \%$ \\
Complete mole & 30 & $58.8 \%$ \\
Term pregnancy & 2 & $3.9 \%$ \\
Total & 51 & $100.0 \%$ \\
\hline
\end{tabular}

Table 2. Histopathology of the studied cases.

\begin{tabular}{lcc}
\hline & Count & $\%$ \\
\hline No endometrial biopsy* & 15 & $29.4 \%$ \\
Choriocarcinoma & 20 & $39.2 \%$ \\
Molar tissues & 10 & $19.6 \%$ \\
Invasive mole & 3 & $5.9 \%$ \\
PSTT** & 3 & $5.9 \%$ \\
Total & 51 & $100.0 \%$ \\
\hline
\end{tabular}

*: Diagnosis of GTN was done by non-normalization of B-hCG after evacuation of hydatidiform mole; PSTT**: Placental site trophoblastic tumor.

Table 3. Sites of distant metastases.

\begin{tabular}{ccc}
\hline Percentage & Number & \\
\hline $62.5 \%$ & 5 & Lungs \\
$12.5 \%$ & 1 & Lungs and brain \\
$12.5 \%$ & 1 & Bone and brain \\
$12.5 \%$ & 1 & Liver \\
$100 \%$ & 8 & Total \\
\hline
\end{tabular}

therapy, where 4 of them achieved remission by this salvage chemotherapy and the other 2 cases did not respond and had hysterectomy. The remaining 3 cases were not given second line chemotherapy; 2 cases had hysterectomy as they refused salvage chemotherapy and they had no distant metastasis while the last case died during the first line therapy after rapid progress with lung and brain metastasis.

Radiotherapy was used in two cases (3.92\%) with brain and/or bone metastasis. Surgical treatment was done in 5 high risk cases (9.8\%). Hysterectomy was done in 4 cases $(7.84 \%)$; as a second line in 2 cases and as a third line in 2 further cases. Hepatic resection was done in one case who presented by internal haemorrhage due to rupture of hepatic metastasis.

The overall survival of GTN cases in our study was $98.04 \%$ and was found to be $100 \%$ for low risk patients.

Table 4 shows the correlation of age, parity, and initial B-hCG to patient response to first-line chemotherapy. As can be seen from this table, patient response was not
Table 4. Correlation of age, parity, and initial B-hCG to patient response to first-line chemotherapy.

\begin{tabular}{cccccc}
\hline & Response & $\mathrm{N}^{*}$ & Mean & S D $^{* *}$ & P value \\
\hline Age & $\begin{array}{c}\text { Responders } \\
\text { Non }\end{array}$ & 37 & 28.73 & 7.85 & 0.953 \\
& $\begin{array}{c}\text { responders } \\
\text { Parity }\end{array}$ & 14 & 28.57 & 9.97 & $(\mathrm{NS})^{* * *}$ \\
& $\begin{array}{c}\text { Responders } \\
\text { Non }\end{array}$ & 37 & 1.38 & 1.497 & \\
responders & 14 & 1.07 & 1.73 & $0.534(\mathrm{NS})$ \\
$\begin{array}{c}\text { Initial } \\
\text { hCG }\end{array}$ & $\begin{array}{c}\text { Responders } \\
\text { Non } \\
\text { responders }\end{array}$ & 37 & 27533.65 & 46849.297 & $\begin{array}{c}0.001 \\
(\text { Sig.) }\end{array}$ \\
\hline
\end{tabular}

$\mathrm{N}^{*}$ : Number of patients; $\mathrm{SD}^{* *}$ : Standard deviation; $(\mathrm{NS})^{* * *}$ : Non significant; (Sig.)****: Significant.

significantly correlated to age and parity but there was significant correlation to initial B- hCG.

Table 5 shows the correlation between type of antecedent pregnancy and patient response to first-line chemotherapy, $\mathrm{P}$ value was 0.74 (non significant).

Table 6 shows the correlation between histopathology and patient response to first-line chemotherapy, again, there was no significant correlation. Table 7 shows the correlation between WHO risk score and patient response to first-line chemotherapy, there was significant correlation $(\mathrm{P}$ value $=0.001)$.

\section{DISCUSSION}

Gestational trophoblastic neoplasia is highly responsive to chemotherapy and prognosis is excellent following treatment, especially in low-risk patients [13]. However, resistance to first line chemotherapy was reported to occur in $33 \%$ of low risk cases and about $10 \%$ of high risk cases $(4,8)$. There is a need to find out the factors that linked to chemo-resistance as well as to reach the ideal treatment strategies for these patients.

This retrospective study was performed through reviewing the records of 51 patients who were diagnosed as gestational trophoblastic neoplasia during the period from 1/1/2006 to 31/12/2010 in the departments of Gynaecology and Clinical Oncology, Mansoura University Hospital, Egypt.

The socio-demographic criteria of GTN patients may differ according to the geographic distribution. The mean age of our cases was 28.7 years which agreed with findings of other authors $[8,12]$ but did not agree with data published by Kaye [14], from Uganda who reported that most of his cases occurred below 20 years or above 35 years. The mean parity of patients in our study was 1.3 which did not agree with Kaye [14] as $68 \%$ of his patients had 5 or more deliveries. This may be explained by different community criteria.

Most of our GTN cases occurred after complete molar 
Table 5. Correlation of type of antecedent pregnancy to patient response to first-line chemotherapy.

\begin{tabular}{cccc}
\hline & \multicolumn{2}{c}{ Response } & Total \\
\cline { 2 - 3 } & Responders & Non responders & \\
\hline Abortion & $6(60 \%)$ & $4(40 \%)$ & 10 \\
Partial mole & $8(88.89 \%)$ & $1(11.11 \%)$ & 9 \\
Complete mole & $22(73.33 \%)$ & $8(26.66 \%)$ & 30 \\
Term pregnancy & $1(50 \%)$ & $1(50 \%)$ & 2 \\
Total & 37 & 14 & 51 \\
\hline
\end{tabular}

$P$ value : 0.47 (non significant).

Table 6. Correlation of histopathology to patient response to first-line chemotherapy.

\begin{tabular}{cccc}
\hline & \multicolumn{2}{c}{ Response } & Total \\
\cline { 2 - 3 } & Responders & $\begin{array}{c}\text { Non } \\
\text { responders }\end{array}$ \\
\hline Endometrial biopsy not done & $13(86.67 \%)$ & $2(13.33 \%)$ & 15 \\
Choriocarcinoma & $13(65 \%)$ & $7(35 \%)$ & 20 \\
Molar tissues & $9(90 \%)$ & $1(10 \%)$ & 10 \\
Invasive mole & $1(33.33 \%)$ & $2(66.67 \%)$ & 3 \\
PSTT & $1(33.33 \%)$ & $2(66.67 \%)$ & 3 \\
Total & 37 & 14 & 51 \\
\hline
\end{tabular}

$P$ value : 0.083 (non significant).

Table 7. Correlation of WHO risk score to patient response to first-line chemotherapy.

\begin{tabular}{cccc}
\hline & \multicolumn{2}{c}{ Response } & \multirow{2}{*}{ Total } \\
\cline { 2 - 3 } & Responders & $\begin{array}{c}\text { Non } \\
\text { responders }\end{array}$ & \\
\hline Low risk (score 0 - 6) & $31(86.11 \%)$ & $5(13.99 \%)$ & 36 \\
High risk (score $\geq 7)$ & $6(40 \%)$ & $9(60 \%)$ & 15 \\
Total & 37 & 14 & 51 \\
\hline
\end{tabular}

P value: 0.001 (Significant).

pregnancies (58.8\%), as can be seen from Table 1, this was in agree with other authors $[15,16]$. Table 2 shows the histopathology of the cases who had been performed uterine curettage. Twenty of thirty-six cases $(55.56 \%)$, were found to be choriocarcinoma this finding was agreed with other authors [17,18]. Distant metastases were shown in Table 3, lung metastasis was the commonest, a similar finding was reported by Kumar et al. [19].

Low risk GTN cases received Methotrexate/folinic acid but there was $15.15 \%$ of patients fail to respond to this monotherapy. Resistance to methotrexate therapy was reported also by other authors $[5,6]$.

High risk cases received MAC combination chemo- therapy, however, $60 \%$ of them were resistant to this protocol. This figure was higher than reports of other authors $[10,12]$, this can be explained by smaller number of patients in the current study.

In this study, we tried to determine whether there were any factors linked to resistance to first line chemotherapy during initial treatment of GTN. Tables 4-7, showed that there was significant correlation of chemotherapy response to initial B-hCG and WHO score ( $\mathrm{p}=$ 0.001 in both). These findings were supported by other author [20,21]. No significant correlation of age, parity, type of antecedent pregnancy, and histopathologic type $(\mathrm{p}=0.95,0.53,0.47 \& 0.83$ respectively). This was supported by the results of Wang et al [22] but did not agree with Bagshawe [20]. This discrepancy may be as result of small number of patients who performed endometrial biopsy (36 cases).

Surgical procedures may be good adjuncts to chemotherapy in properly selected cases as the majority of women with GTN are young and wish to preserve their fertility. Hysterectomy was done in 4 high-risk cases who failed to respond to first line chemotherapy as a second or third line therapy. This findings was also supported by other authors $[12,17,23]$.

We have to report that this retrospective study represented a single center experience and had relatively small number of cases. A large multicenter prospective trial is recommended.

\section{CONCLUSIONS}

Low risk GTN cases were found to be resistant to methotrexate monotherapy in $15.15 \%$, these cases received etoposide or cisplatinum/etoposide as a second-line therapy. High risk GTN cases received MAC combination but $60 \%$ were resistant and needed second line combination chemotherapy or hysterectomy. WHO score and initial B-hCG were significantly correlated to resistance to first-line chemotherapy.

\section{REFERENCES}

[1] Disaia, P.J. and Creasman, W.T. (2007) Gestational trophoblastic neoplasia, 7th Edition. Clinical Gynaecologic Oncology, Mosby Inc, I, 201-233.

[2] Berkowitz, R.S. and Goldstein, D.P.(2009) Current management of gestational trophoblastic diseases. Gynecologic Oncology, 112, 654-662. doi:10.1016/j.ygyno.2008.09.005

[3] Moodley, M., Tunkyi, K. and Moodley J. (2003) Gestational trophoblastic syndrome: An audit of 112 patients. A South African experience. International Journal of Gynecological Cancer, 13, 234-239.

[4] Bagshawe, K.D., Dent, J., Newlands, E.S., Begent, R.H.J. and Rustin, G.J.S. (1989) The role of low-dose methotrexate and folinic acid in gestational trophoblastic tumors. British Journal of Obstetrics and Gynecology, 96, 
795-802. doi:10.1111/j.1471-0528.1989.tb03318.x

[5] Homesley, H.D., Blessing, J.A., Rottenmaier, M., Capizzi, R.L., Major, F.J. and Twiggs, L.B.(1988) Weekly intramuscular methotrexate for nonmetastatic gestational trophoblastic disease. Obstetrics and Gynecology, 72, 413418.

[6] McNeish, I.A., Strickland, S., Holden, L., et al. (2002) Low-risk persistent gestational trophoblastic disease: Out-come after initial treatment with low-dose methotrexate and folinic acid from 1992-2000. Journal of Clinical Oncology, 20, 1838-1844. doi:10.1200/JCO.2002.07.166

[7] Bower, M., Newlands, E.S., Holden, L., et al. (1997) EMA/CO for high-risk gestational trophoblastic tumours: Results from a cohort of 272 patients. Journal of Clinical Oncology, 15, 2636-2643.

[8] Newlands, E.S., Mulholland, P.J. and Holden, 1., et al. (2000) Etoposide and cisplatin/etoposide, methotrexate, and actinomycin D (EMA) chemotherapy for patients with high-risk gestational trophoblastic tumors refractory to EMA/cyclophosphamide and vincristine chemotherapy and patients presenting with metastatic placental site trophoblastic tumors. Journal of Clinical Oncology, 18, 854-859.

[9] El-Lamie, I.K., El Sayed, H.M., Badawie, A.G., Bayomi, W.A., El-Ghazaly, H.A., Khalaf-Allah, A.E., El-Mahallawy, M.N. and El-Lamie, K.I. (2006) Evolution of treatment of high-risk metastatic gestational trophoblastic tumors: Ain shams university experience. International Journal of Gynecological Cancer, 16, 866-874.

[10] Deng, L., Yan, X., Zhang, J. and Wu, T. (2009) Combination chemotherapy for high-risk gestational trophoblastic tumour. Cochrane Database of System Reviews, 15, CD-005196.

[11] Patel, S.M. and Desai, A. (2010) Management of drug resistant gestational trophoblastic neoplasia. The Journal of Reproductive Medicine, 55, 296-300.

[12] Zhou, Y., Feng, F.Z., Xiang, Y. and Wan, X.R. (2010) Clinical analysis of patients with relapsed and chemoresistant gestational trophoblastic neoplasia. Chinese Journal of Obstertrics and Gynecology, 45, 804-807.

[13] Ngan, S. and Seckl, M.J. (2007) Gestational trophoblastic neoplasia management: An update. Current Opinion in Oncology, 19, 486-491.
doi:10.1097/CCO.0b013e3282dc94e5

[14] Kaye, D.K. (2002) Gestational trophoblastic disease following complete hydatidiform modle in Mulago Hospital, Kampala, Uganda. African Health Science, 2, 4751.

[15] Sebire, N.J. and Seckl, M.J. (2008) Gestational trophoblastic disease: Current management of hydatidiform mole. British Medicine Journal, 337, 1193. doi:10.1136/bmj.a1193

[16] Kuyumcuoglu, U., Guzel, A., Erdemoglu, M. and Celik Y. (2011) Risk factors for persistent gestational trophoblastic neoplasia. Journal of Experimental Therapeutics and Oncology, 9, 81-84.

[17] Pongsaranantakul, S. and Kietpeerakool, C. (2009) Hystere-ctomy in gestational trophoblastic neoplasia: chiang mai university hospital experience. Asian Pacific Journal of Cancer Prevention, 10, 311-314.

[18] Sebire, N.J. and Lindsay, I. (2010) Current Issues in the his- topathology of gestational trophoblastic tumors. Fetal and Pediatric Pathology, 29, 30-44. doi:10.3109/15513810903266120

[19] Kumar, J., Ilancheran, A. and Ratnam, S. (1988) Pulmonary metastases in gestational trophoblastic disease: A review of 97 cases. British Journal of Obstetrics and Gynaecology, 95, 70-74. doi:10.1111/j.1471-0528.1988.tb06482.x

[20] Bagshawe, K.D. (1976) Risk and prognostic factors in trophoblastic neoplasia. Cancer, 38, 1373-1385. doi:10.1002/1097-0142(197609)38:3<1373::AID-CNCR 2820380342>3.0.CO;2-E

[21] Deligdisch, L., Driscoll, S.G. and Goldstein, D.P. (1978) Gestational trophoblastic neoplasms: Morphologic correlates of therapeutic response. American Journal of Obstetrics and Gynecology, 130, 801-806.

[22] Wang, J., Short, D., Sebire, N., Lindsay, I., Newlands, E., Schmid, P., Savage, P. and Seckl, M (2008) Salvage chemotherapy of relapsed or high-risk gestational trophoblastic neoplasia (GTN) with paclitaxel/cisplatin alternating with paclitaxel/etoposide (TP/TE). Annals of Oncology, 19, 1578-1583. doi:10.1093/annonc/mdn181

[23] Fülöp, V., Szigetvŕi, I., Szepesi, J., Török, M. and Berkowitz, R.S. (2008) Diagnosis and treatment of highrisk metastatic gestational trophoblastic neoplasia in Hungary. Journal of Reproductive Medicine, 53, 541-546. 